\title{
Clinicopathological significance of ataxia telangiectasia-mutated (ATM) kinase and ataxia telangiectasia-mutated and Rad3-related (ATR) kinase in MYC overexpressed breast cancers
}

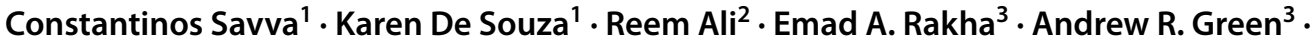 \\ Srinivasan Madhusudan ${ }^{1,2}$ (iD
}

Received: 13 December 2018 / Accepted: 18 December 2018 / Published online: 12 February 2019

(c) The Author(s) 2019

\begin{abstract}
Purpose MYC transcription factor has critical roles in cell growth, proliferation, metabolism, differentiation, transformation and angiogenesis. MYC overexpression is seen in about $15 \%$ of breast cancers and linked to aggressive phenotypes. MYC overexpression also induces oxidative stress and replication stress in cells. ATM signalling and ATR-mediated signalling are critical for MYC-induced DNA damage response. Whether ATM and ATR expressions influence clinical outcomes in MYC overexpressed breast cancers is unknown.

Methods We investigated ATM, ATR and MYC at the transcriptional level [Molecular Taxonomy of Breast Cancer International Consortium cohort $(n=1950)$ ] and at the protein level in the Nottingham series comprising 1650 breast tumours. We correlated ATM, ATR and MYC expressions to clinicopathological features and survival outcomes.

Results In MYC over expressed tumours, high ATR or low ATM levels were associated with aggressive breast cancer features such as higher tumour grade, de-differentiation, pleomorphism, high mitotic index, high-risk Nottingham Prognostic Index, triple negative and basal-like breast cancers (all adjusted $p$ values $<0.05$ ). Tumours with low ATM or high ATR levels in conjunction with MYC overexpression also have worse overall breast cancer-specific survival (BCSS) $(p$ value $<0.05)$. Conclusions We conclude that ATR/ATM-directed stratification and personalisation of therapy may be feasible in MYC overexpressed breast cancer.
\end{abstract}

Keywords ATM $\cdot$ ATR $\cdot$ MYC $\cdot$ Breast cancer

\section{Background}

Constantinos Savva and Karen De Souza contributed equally to this work.

Electronic supplementary material The online version of this article (https://doi.org/10.1007/s10549-018-05113-8) contains supplementary material, which is available to authorized users.

Andrew R. Green

Andrew.green@nottingham.ac.uk

$\triangle$ Srinivasan Madhusudan

srinivasan.madhusudan@nottingham.ac.uk

1 Department of Oncology, Nottingham University Hospitals, Nottingham NG5 1PB, UK

2 Translational Oncology, Nottingham Breast Cancer Research Centre, Division of Cancer and Stem Cells, Academic Unit of Oncology, School of Medicine, University of Nottingham, Nottingham NG51 PB, UK
The c-MYC transcription factor has critical roles in cell growth, proliferation, metabolism, differentiation, transformation and angiogenesis. Overexpression of c-MYC (henceforth MYC) is frequently observed in several solid tumours implying a critical role in tumorigenesis and progression.

Department of Pathology, Nottingham Breast Cancer Research Centre, Division of Cancer and Stem Cells, School of Medicine, University of Nottingham, Nottingham NG5 1PB, UK 
In addition, MYC overexpression is linked to resistance to chemotherapy and radiotherapy $[5,12,15,17,19,27,35$, 37]. In breast cancers, MYC gene amplification (15\%), MYC mRNA overexpression (22-35\%) and MYC protein overexpression (40\%) have been reported. MYC overexpression has been linked to specific subtypes of aggressive breast cancers $[7,16,21,38]$.

Overexpression of MYC and resultant oncogenic stress can induce DNA damage and impact genomic stability. MYC-induced oxidative stress leads to oxidative DNA base damage [6]. In addition, MYC overexpression can also promote replication stress in cells [17]. Ataxia telangiectasia-mutated kinase (ATM) and ataxia telangiectasia and Rad3-related kinase (ATR) are critical for c-MYC-induced DNA damage response [18, 24, 32]. ATR is activated and recruited to sites of single-stranded (ss) double-stranded (ds) DNA damage, during nucleotide excision repair, at resected double-strand breaks and stalled replication forks. Activated ATR in turn phosphorylates Chk1 at $\mathrm{Ser}^{345}$ and $\mathrm{Ser}^{317}$, as well as several other target proteins involved in DNA repair and cell cycle progression [18, 24, 32]. ATM kinase is activated in response to DNA damage [34]. A key substrate of ATM is Chk2 whose phosphorylation at Thr68 results in activation and phosphorylation of a several proteins involved in DNA repair, recombination, cell cycle progression and apoptosis [34].

In the current study, we comprehensive investigated ATM, ATR and MYC expressions at the transcriptional levels $(n=1950)$ and at the protein level $(n=1650)$ breast tumours. We show that ATM and ATR levels have clinicopathological, predictive and prognostic significance in MYC overexpressed breast cancer.

\section{Methods}

\section{Tissue culture and western blotting}

Cell lines were purchased from American Type Culture Collection (ATCC, Manassas, USA). MDA-MB-231 and MDA-MB-468 cells were cultured in minimum essential amino acids medium supplemented with $1 \%$ L-glutamine and $1 \%$ non-essential amino acids. T47D cells were cultured in Dulbecco's Modified Eagle's medium. MCF-7 cells were grown in RPMI medium. All media were supplemented with $10 \%$ FBS and $1 \%$ penicillin streptomycin. Protein samples were prepared by lysing cells in RIPA buffer (Sigma-Aldrich) containing protease inhibitor (Sigma) and phosphatase inhibitor cocktail 1 and 2 (Sigma). Samples were run on SDS-PAGE gel (4-12\%) bis-tris. Antibodies used were anti-MYC antibody (abcam, clone 9E10), ATM antibody (abcam clone Y170) and ATR antibody (cell signalling cat.no 2790S). Protein detection and quantification were determined by scanning the membranes on LicorOdyssey's Scanner (Licor, Biosciences) at the predefined intensity fluorescence.

\section{MYC, ATM and ATR mRNA expressions in breast cancer}

$M Y C, A T M$ and ATR mRNA expressions were investigated in METABRIC (Molecular Taxonomy of Breast Cancer International Consortium) cohort. The METABRIC study protocol, detailing the molecular profiling methodology in a cohort of 1977 breast cancer samples is described by Curtis et al. [14]. Patient demographics are summarised in Supplementary Table $\mathrm{S} 1$ of supporting information. ER-positive and/or lymph node-negative patients did not receive adjuvant chemotherapy. ER-negative and/or lymph node-positive patients received adjuvant chemotherapy. For this cohort, the mRNA expression was hybridised to Illumina HT-12 v3 platform (Bead Arrays), and the data were pre-processed and normalised as described previously. Samples were classified into the intrinsic subtypes based on the PAM50 gene list. A description of the normalisation, segmentation and statistical analyses was previously described [14]. Real-time RTqPCR was performed on the ABI Prism 7900HT sequence detection system (Applied Biosystems) using SYBR1 Green reporter. All the samples were analysed as triplicates. The Chi-square test was used for testing association between categorical variables, and a multivariate Cox model was fitted to the data using as endpoint breast cancer-specific death. X-tile (Version 3.6.1) was used to identify a cut-off in gene expression values such that the resulting subgroups had significantly different survival courses.

\section{MYC, ATM and ATR protein expressions in breast cancer}

The study was performed in a consecutive series of 1650 patients with primary invasive breast carcinomas who were diagnosed between 1986 and 1999 and entered into the Nottingham Tenovus Primary Breast Carcinoma series. Patient demographics are summarised in Supplementary Table S2. This is a well-characterised series of patients with long-term follow-up that have been investigated in a wide range of biomarker studies $[1,2,21]$. All patients were treated in a uniform way in a single institution with standard surgery (mastectomy or wide local excision), followed by Radiotherapy. Prior to 1989, patients did not receive systemic adjuvant treatment (AT). After 1989, AT was scheduled based on prognostic and predictive factor status, including Nottingham Prognostic Index (NPI), oestrogen receptor- $\alpha$ $($ ER- $\alpha$ ) status, and menopausal status. Patients with NPI scores of $<3.4$ (low risk) did not receive AT. In premenopausal patients with NPI scores of $\geq 3.4$ (high risk), 
classical Cyclophosphamide, Methotrexate, and 5-Fluorouracil (CMF), chemotherapy was given; patients with ER- $\alpha$-positive tumours were also offered endocrine therapy. Postmenopausal patients with NPI scores of $\geq 3.4$ and ER- $\alpha$ positivity were offered endocrine therapy, while ER- $\alpha$ negative patients received classical CMF chemotherapy. Median follow-up was 111 months (range 1-233 months). Survival data, including breast cancer-specific survival (BCSS), disease-free survival (DFS), and development of loco-regional and distant metastases (DM), were maintained on a prospective basis. DFS was defined as the number of months from diagnosis to the occurrence of local recurrence, local lymph node (LN) relapse or DM relapse. Breast cancer-specific survival (BCSS) was defined as the number of months from diagnosis to the occurrence of BC-related death. Local recurrence-free survival (LRS) was defined as the number of months from diagnosis to the occurrence of local recurrence. DM-free survival was defined as the number of months from diagnosis to the occurrence of DM relapse. Survival was censored if the patient was still alive at the time of analysis, lost to follow-up, or died from other causes.

Tumour Marker Prognostic Studies (REMARK) criteria, recommended by McShane et al. [28], were followed throughout this study. Ethical approval was obtained from the Nottingham Research Ethics Committee (C202313).

\section{Tissue microarrays (TMAs) and immunohistochemistry (IHC)}

Tumours were arrayed in tissue microarrays (TMAs) constructed with $0.6 \mathrm{~mm}$ cores sampled from the periphery of the tumours. The TMAs were immunohistochemically profiled for MYC, ATM and ATR and other biological antibodies as previously described [1, 2, 21]. Immunohistochemical staining was performed using the Thermo Scientific Shandon Sequenza chamber system (REF: 72110017), in combination with the Novolink Max Polymer Detection System (RE7280-K: 1250 tests), and the Leica Bond Primary Antibody Diluent (AR9352), each used according to the manufacturer's instructions (Leica Microsystems). Leica Autostainer XL machine was used to dewax and rehydrate the slides. Pre-treatment antigen retrieval was performed on the TMA sections using sodium citrate buffer ( $\mathrm{pH}$ 6.0) and heated for $20 \mathrm{~min}$ at $95^{\circ} \mathrm{C}$ in a microwave (Whirpool JT359 Jet Chef 1000W). A set of slides were incubated for $18 \mathrm{~h}$ at $4{ }^{\circ} \mathrm{C}$ with the primary mouse monoclonal anti-ATM antibody, clone Y170 (Ab32420, Abcam, Cambridge, UK), at a dilution of 1:100. A set of slides were incubated for $18 \mathrm{~h}$ at $4{ }^{\circ} \mathrm{C}$ with the primary mouse monoclonal anti-ATR antibody, clone 1E9 (H00000545-M03, Novus Biologicals, Cambridge, UK), at a dilution of 1:20. A set of slides were incubated for $45 \mathrm{~min}$ at $4{ }^{\circ} \mathrm{C}$ with mouse monoclonal primary antibody for c-MYC (Clone 9E100; Abcam Ltd) at a dilution of 1: 100 .

\section{Evaluation of immune staining}

Whole field inspection of the core was scored and intensities of nuclear staining were grouped as follows: $0=$ no staining, $1=$ weak staining, $2=$ moderate staining, $3=$ strong staining. The percentage of each category was estimated $(0-100 \%)$. $\mathrm{H}$-score (range 0-300) was calculated by multiplying intensity of staining and percentage staining. X-tile (version 3.6.1, Yale University, USA) was used to identify a cut-off for ATM protein expression. The percentage of positive cells was used, with a cut-off of $<25 \%$ cells being classed as low, and $\geq 25 \%$ as high for ATM protein level. X-tile (version 3.6.1, Yale University, USA) was used to identify a cut-off for protein expression. H-score of $\geq 60$ was taken as the cut-off for high ATR expression. Assessment of MYC staining was estimated subjectively on intensity corresponding to negative, weak, moderate and strong nuclear and/or cytoplasmic staining. Dichotomisation of c-MYC protein expression was based on the mean resulting in negative/ weak (MYC negative) and moderate/strong (MYC positive) groups, which were selected prior to analysis.

\section{Statistical analysis}

Data analysis was performed using SPSS (SPSS, version 17 Chicago, IL). Where appropriate, Pearson's Chi-square, Fisher's exact, Student's t and ANOVA one-way tests were used. Cumulative survival probabilities were estimated using the Kaplan-Meier method, and differences between survival rates were tested for significance using the log-rank test. Multivariate analysis for survival was performed using the Cox proportional hazard model. The proportional hazards assumption was tested using standard log-log plots. Hazard ratios (HR) and $95 \%$ confidence intervals $(95 \% \mathrm{CI})$ were estimated for each variable. All tests were two-sided with a $95 \% \mathrm{CI}$ and a $p$ value $<0.05$ considered significant. For multiple comparisons, $\mathrm{p}$ values were adjusted according to Benjamini-Hochberg method [23].

\section{Results}

We initially performed western blots in a panel of breast cancer cell lines to confirm the specificity of antibodies for IHC in the current study. As shown in Fig. 1a, the antibodies tested were not only specific but also demonstrated a spectrum of protein expression levels across various breast cancer cell lines (Supplementary Fig. S1). We then proceeded to investigate MYC, ATR and ATM protein levels in clinical breast carcinoma samples. 

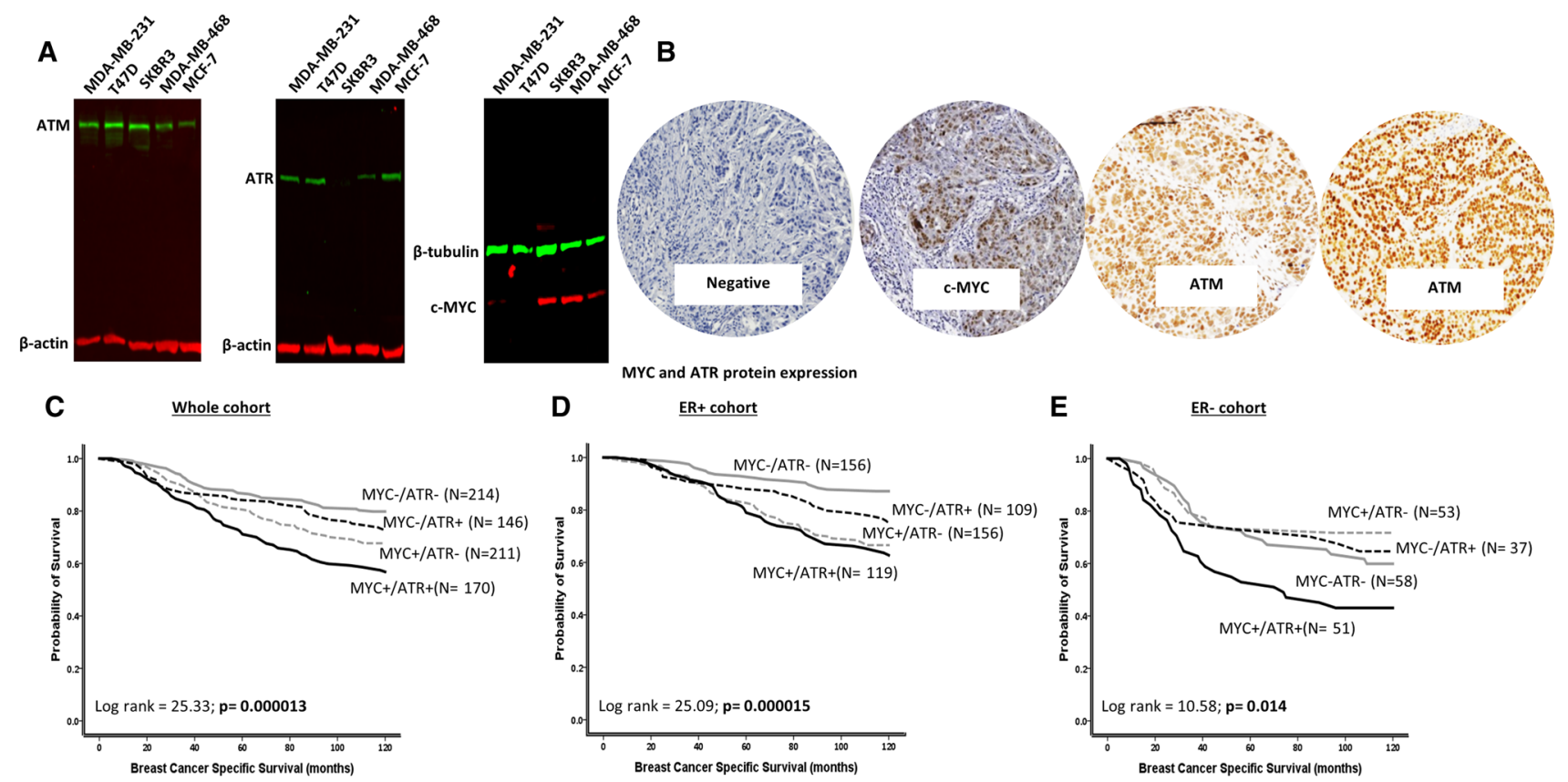

D $\underline{E R+\text { cohort }}$
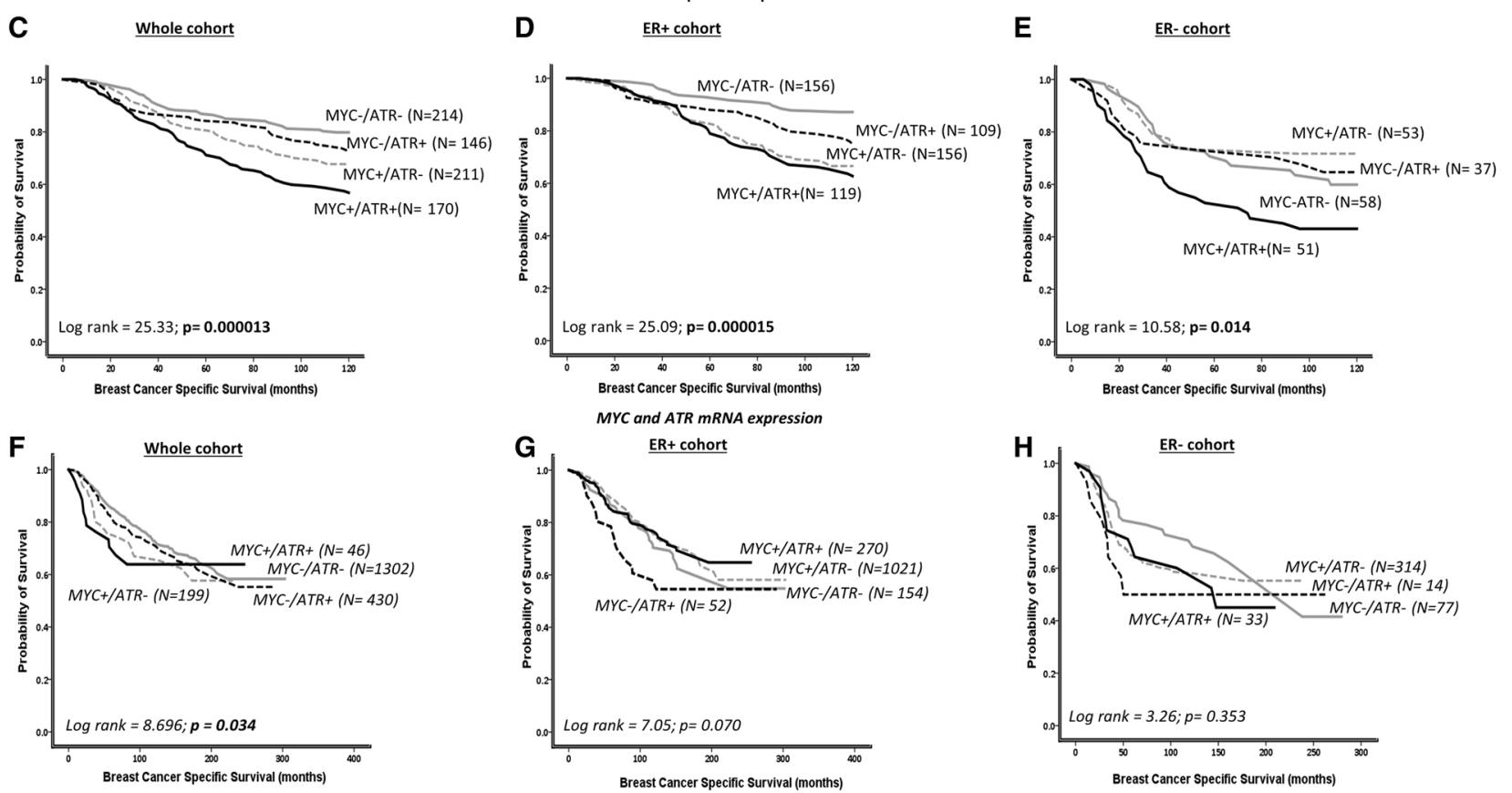

Fig. 1 a Western blot of c-MYC, ATM and ATR expressions in breast cancer cell lines. b Microphotograph of MYC-negative and MYC-positive breast cancers. $\mathbf{c}-\mathbf{h}$ Kaplan-Meier curves showing BCSS in MYC and ATR co-expression at protein and mRNA levels

\section{High ATR promotes aggressive phenotypes in MYC overexpressed breast cancers}

A total of 793 tumours were suitable for ATR and MYC protein co-expression analyses (Fig. 1b). Tumours with high MYC and high ATR expressions were significantly associated with vascular invasion, higher tumour grade, pleomorphism, high mitotic index and high-risk Nottingham Prognostic Index (NPI) (all adjusted $p$ values $\leq 0.01$ ) (Table 1).

At the transcriptomic level (Table 2), tumours with high $M Y C$ mRNA and high ATR mRNA expression were also associated with higher tumour grade, high-risk Nottingham Prognostic Index (NPI), ER-, PR-, Genefu subtype (ER-/Her-2-), triple negative and PAM50. Basal phenotypes (all adjusted $p$ values $\leq 0.01$ ). Interestingly, Genufu subtype (ER+/HER-2-/low proliferation),
Her-2+, PAM50.Her-2 subtype and PAM50.Luminal A subtype were more common in tumours with low $M Y C$ mRNA and low ATR mRNA expressions (all adjusted $p$ values $\leq 0.01$ ).

We then investigated the prognostic significance of MYC-ATR co-expression in breast cancers. In the whole cohort, as shown in Fig. 1c, f, patients with MYC overexpressed tumours and high ATR protein or mRNA expression had a worse overall breast cancer-specific survival (BCSS) $(p<0.001)$. In ER + breast cancer, similarly, MYC overexpressed tumours with high ATR levels are associated with worse survival $(p<0.001)$ (Fig. 1d) including in patients who received endocrine therapy (Supplementary Figs. S2B and S2F). In ER- tumours, MYC overexpressed tumours with high ATR protein levels had the worst survival (Fig. 2e). Together the data show that MYC-ATR coexpression has prognostic significance in breast cancers. 
Table 1 ATR and MYC protein co-expression in Sporadic Breast Cancer

\begin{tabular}{|c|c|c|c|c|c|c|}
\hline \multirow[t]{3}{*}{ Variable } & \multicolumn{4}{|c|}{ ATR and MYC protein co-expression } & \multirow[t]{3}{*}{$p$ value } & \multirow[t]{3}{*}{ Adjusted $p$ value* } \\
\hline & MYC-/ATR- & MYC-/ATR+ & MYC+/ATR- & MYC+/ATR+ & & \\
\hline & $N(\%)$ & $N(\%)$ & $N(\%)$ & $N(\%)$ & & \\
\hline \multicolumn{7}{|l|}{ Tumour size (cm) } \\
\hline$\leq 2.0$ & $115(50.0)$ & $59(38.1)$ & $110(49.1)$ & $73(39.7)$ & \multirow[t]{2}{*}{0.03} & \multirow[t]{2}{*}{0.053} \\
\hline$>2.0$ & $115(50.0)$ & 96 (61.9) & $114(50.9)$ & $111(60.3)$ & & \\
\hline \multicolumn{7}{|l|}{ Vascular invasion } \\
\hline Negative & $166(72.5)$ & $94(60.6)$ & $152(68.8)$ & $106(57.6)$ & \multirow[t]{2}{*}{0.005} & \multirow[t]{2}{*}{0.013} \\
\hline Positive & $63(27.5)$ & $61(39.4)$ & $69(31.2)$ & $78(42.4)$ & & \\
\hline \multicolumn{7}{|l|}{ Tumour grade ${ }^{\mathrm{a}}$} \\
\hline G1 & $48(20.9)$ & $18(11.8)$ & $40(17.9)$ & $15(8.2)$ & \multirow[t]{3}{*}{0.001} & \multirow[t]{3}{*}{0.004} \\
\hline G2 & $78(33.9)$ & $42(27.4)$ & $70(31.4)$ & $51(27.9)$ & & \\
\hline G3 & $104(45.2)$ & $93(60.8)$ & $113(50.7)$ & $117(63.9)$ & & \\
\hline \multicolumn{7}{|l|}{ Tumour type } \\
\hline Ductal (incl mixed) & $192(83.6)$ & $134(86.4)$ & $195(87.1)$ & $169(91.8)$ & \multirow[t]{5}{*}{0.076} & \multirow[t]{5}{*}{0.121} \\
\hline Lobular & $20(8.7)$ & $15(9.7)$ & $13(5.8)$ & $7(3.8)$ & & \\
\hline Medullary-like & $7(3.0)$ & $0(0.0)$ & $10(4.5)$ & $6(3.3)$ & & \\
\hline Miscellaneous & $1(0.4)$ & $0(0.0)$ & $0(0.0)$ & $0(0.0)$ & & \\
\hline Special type & $10(4.3)$ & $6(3.9)$ & $6(2.6)$ & $2(1.1)$ & & \\
\hline \multicolumn{7}{|l|}{ Tubules } \\
\hline 1 & $14(6.4)$ & $5(3.2)$ & $9(4.3)$ & $5(2.7)$ & \multirow[t]{3}{*}{0.583} & \multirow[t]{3}{*}{0.717} \\
\hline 2 & $75(34.4)$ & $48(31.4)$ & $72(34.1)$ & $65(35.5)$ & & \\
\hline 3 & $129(59.2)$ & $100(65.4)$ & $130(61.6)$ & $113(61.8)$ & & \\
\hline \multicolumn{7}{|l|}{ Pleomorphism } \\
\hline 1 & $2(0.9)$ & $1(0.6)$ & $6(2.9)$ & $1(0.5)$ & \multirow[t]{3}{*}{0.003} & 0.0096 \\
\hline 2 & $93(42.7)$ & $61(39.9)$ & $78(37.3)$ & $47(25.7)$ & & \\
\hline 3 & $123(56.4)$ & $91(59.5)$ & $125(59.8)$ & $135(73.8)$ & & \\
\hline Mitosis & & & & & & \\
\hline 1 & $92(42.2)$ & $40(26.2)$ & $66(31.3)$ & $38(20.8)$ & 0.00012 & 0.00064 \\
\hline 2 & $36(16.5)$ & $21(13.7)$ & $40(18.9)$ & $35(19.1)$ & & \\
\hline 3 & $90(41.3)$ & $92(60.1)$ & $105(49.8)$ & $110(60.1)$ & & \\
\hline NPI group & & & & & & \\
\hline GPG & $79(34.3)$ & $35(22.6)$ & $71(31.7)$ & $33(17.9)$ & $<0.00001$ & $<0.00001$ \\
\hline MPG & $128(55.7)$ & $83(53.5)$ & $125(55.8)$ & $96(52.2)$ & & \\
\hline PPG & $23(10.0)$ & $37(23.9)$ & $28(12.5)$ & $55(29.9)$ & & \\
\hline ER status & & & & & & \\
\hline Negative & $63(27.4)$ & $38(24.5)$ & $58(26.2)$ & $51(27.7)$ & 0.908 & 0.968 \\
\hline Positive & $167(72.6)$ & $117(75.5)$ & $163(73.8)$ & $133(72.3)$ & & \\
\hline PR status & & & & & & \\
\hline Negative & $105(45.9)$ & $64(42.4)$ & $98(44.7)$ & $77(42.3)$ & 0.864 & 0.987 \\
\hline Positive & $124(54.1)$ & 87 (57.6) & $121(55.3)$ & $105(57.7)$ & & \\
\hline HER2 status & & & & & & \\
\hline Negative & $187(82.7)$ & $122(81.3)$ & $194(87.0)$ & $151(82.5)$ & 0.437 & 0.582 \\
\hline Positive & $39(17.3)$ & $28(18.7)$ & $29(13.0)$ & $32(17.5)$ & & \\
\hline Triple negative & & & & & & \\
\hline Non-triple negative & $188(82.5)$ & $132(86.8)$ & 175 (79.5) & $144(78.3)$ & 0.183 & 0.266 \\
\hline Triple negative & $40(17.5)$ & $20(13.2)$ & $45(20.5)$ & $40(21.7)$ & & \\
\hline Basal phenotype & & & & & & \\
\hline Negative & $175(76.8)$ & $121(78.1)$ & $149(66.8)$ & $127(69.0)$ & 0.027 & 0.054 \\
\hline Positive & $53(23.2)$ & 34 (21.9) & $74(33.2)$ & $57(31.0)$ & & \\
\hline
\end{tabular}

Bold statistically significant; NPI Nottingham Prognostic Index, GPG Good Prognosis Group, MPG Moderate Prognosis Group, PPG Poor Prognosis Group, $H E R 2$ human epidermal growth factor 2, $E R$ oestrogen receptor, $P R$ progesterone receptor

*Adjusted $P$ value - Benjamini and Hochberg false discovery rate

${ }^{\mathrm{a}}$ Grade as defined by Nottingham Grading System 
Table 2 ATR and MYC mRNA co-expression in sporadic breast cancer

\begin{tabular}{|c|c|c|c|c|c|c|}
\hline \multirow[t]{3}{*}{ Variable } & \multicolumn{4}{|c|}{$A T R$ and $M Y C$ mRNA co-expression } & \multirow[t]{3}{*}{$p$ value } & \multirow[t]{3}{*}{ Adjusted $p$ value* } \\
\hline & MYC-/ATR- & MYC-/ATR+ & MYC+/ATR- & MYC+/ATR+ & & \\
\hline & $N(\%)$ & $N(\%)$ & $N(\%)$ & $N(\%)$ & & \\
\hline \multicolumn{7}{|l|}{ Tumour size $(\mathrm{cm})$} \\
\hline $\mathrm{T} 1 \mathrm{a}+\mathrm{b}(1.0)$ & $58(4.5)$ & $24(5.6)$ & $9(4.6)$ & $1(2.2)$ & 0.333 & 0.428 \\
\hline $\mathrm{T} 1 \mathrm{c}(>1.0-2.0)$ & $527(40.9)$ & $156(36.6)$ & $64(32.5)$ & $19(42.2)$ & & \\
\hline $\mathrm{T} 2(>2.0-5)$ & $648(50.3)$ & $220(51.6)$ & $111(56.3)$ & $22(48.9)$ & & \\
\hline $\mathrm{T} 3(>5)$ & $56(4.3)$ & $26(6.1)$ & $13(6.6)$ & $3(6.7)$ & & \\
\hline \multicolumn{7}{|l|}{ Lymph node stage } \\
\hline Negative & $686(52.8)$ & $232(54.1)$ & $91(46.4)$ & $26(56.5)$ & 0.612 & 0.718 \\
\hline Positive (1-3) & $210(16.2)$ & $65(15.2)$ & $34(17.3)$ & $5(10.9)$ & & \\
\hline Positive $(>3)$ & $404(31.1)$ & $132(30.8)$ & $71(36.2)$ & $15(32.6)$ & & \\
\hline \multicolumn{7}{|l|}{ Grade $^{\mathrm{a}}$} \\
\hline G1 & $120(9.7)$ & $37(9.0)$ & $11(5.6)$ & $1(2.3)$ & 0.00018 & 0.00048 \\
\hline $\mathrm{G} 2$ & $524(42.2)$ & $176(42.9)$ & $57(29.2)$ & $13(30.2)$ & & \\
\hline G3 & $597(48.1)$ & $197(48.0)$ & $127(65.1)$ & $29(67.4)$ & & \\
\hline \multicolumn{7}{|l|}{ NPI } \\
\hline$\leq 3.4$ & $274(22.2)$ & $84(20.7)$ & $25(13.2)$ & $5(11.9)$ & 0.016 & 0.0288 \\
\hline$>3.4$ & $958(77.8)$ & $322(79.3)$ & $165(86.8)$ & $37(88.1)$ & & \\
\hline \multicolumn{7}{|l|}{ HER 2 overexpression } \\
\hline No & $1119(85.9)$ & $382(88.8)$ & $187(94.0)$ & $44(95.7)$ & 0.003 & 0.00675 \\
\hline Yes & $183(14.1)$ & $48(11.2)$ & $12(6.0)$ & $2(4.3)$ & & \\
\hline \multicolumn{7}{|l|}{ ER } \\
\hline Negative & $270(20.7)$ & $92(21.4)$ & $84(42.2)$ & $24(52.2)$ & $<0.00001$ & $<0.00001$ \\
\hline Positive & $1032(79.3)$ & 338 (78.6) & $115(57.8)$ & $22(47.8)$ & & \\
\hline \multicolumn{7}{|l|}{ PR } \\
\hline Negative & $597(45.9)$ & $194(45.1)$ & $116(58.3)$ & $29(63.0)$ & 0.001 & 0.00245 \\
\hline Positive & $705(54.1)$ & $236(54.9)$ & $83(41.7)$ & $17(37.0)$ & & \\
\hline \multicolumn{7}{|l|}{ Triple negative } \\
\hline No & 1137(87.3) & $371(86.3)$ & $126(63.3)$ & $26(56.5)$ & $<0.00001$ & $<0.00001$ \\
\hline Yes & $165(12.7)$ & 59 (13.7) & $73(36.7)$ & $20(43.5)$ & & \\
\hline \multicolumn{7}{|l|}{ Genefu subtype } \\
\hline ER-/Her-2 negative & $77(11.5)$ & $30(14.6)$ & $35(32.4)$ & $8(57.1)$ & $<0.00001$ & $<0.00001$ \\
\hline ER+/Her-2 negative/high proliferation & $231(34.6)$ & $87(42.4)$ & $45(41.7)$ & $3(21.4)$ & 0.083 & 0.124 \\
\hline ER+/Her-2 negative/low proliferation & $280(42.0)$ & $65(31.7)$ & $21(19.4)$ & $2(14.3)$ & 0.00001 & 0.00002 \\
\hline Her-2 positive & $79(11.8)$ & $23(11.2)$ & $7(6.5)$ & $1(7.1)$ & 0.401 & 0.492 \\
\hline \multicolumn{7}{|l|}{ PAM50 subtype } \\
\hline PAM50.Her2 & $166(14.2)$ & $66(16.4)$ & $6(3.7)$ & $0(0.0)$ & 0.00005 & 0.000156 \\
\hline PAM50.Basal & $173(14.8)$ & $54(13.4)$ & $77(47.0)$ & $26(74.3)$ & $<0.00001$ & $<0.00001$ \\
\hline PAM50.LumA & $509(43.5)$ & $166(41.2)$ & $38(23.2)$ & $2(5.7)$ & $<0.00001$ & $<0.00001$ \\
\hline PAM50.LumB & $322(27.5)$ & $117(29.0)$ & $43(26.2)$ & $7(20.0)$ & 0.663 & 0.745 \\
\hline
\end{tabular}

Bold statistically significant; NPI Nottingham Prognostic Index, $H E R 2$ human epidermal growth factor 2, $E R$ oestrogen receptor, $P R$ progesterone receptor

*Adjusted $P$ value-Benjamini and Hochberg false discovery rate

${ }^{a}$ Grade as defined by Nottingham Grading System 
MYC and ATM protein expression
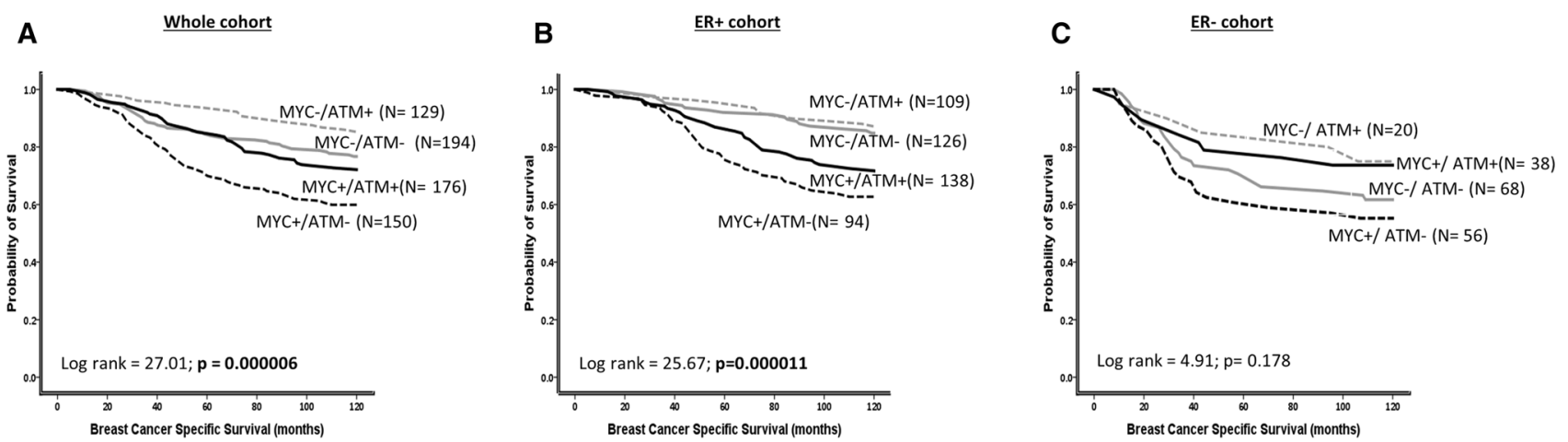

MYC and ATM mRNA expression
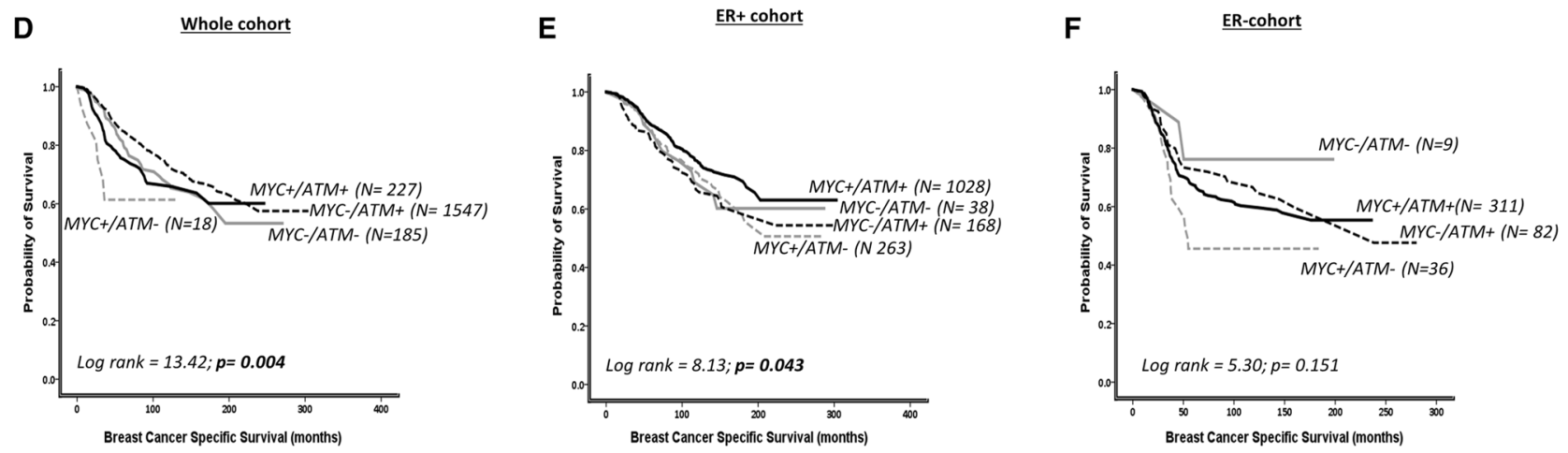

Fig. 2 Kaplan-Meier curves showing BCSS in MYC and ATM co-expression at protein and mRNA levels

\section{Low ATM promotes aggressive phenotypes in MYC overexpressed breast cancers}

A total of 696 tumours were suitable for ATM and MYC protein expression analyses (Fig. 1b) (Table 3). Tumours with high MYC and low ATM expressions were significantly associated with higher tumour grade, tumour type, pleomorphism, high mitotic index, ER-, PR-, triple negative, basal phenotypes and high-risk Nottingham Prognostic Index (NPI) (all adjusted $p$ values $\leq 0.001$ ).

At the transcriptomic level (Table 4), tumours with high $M Y C$ mRNA and low ATM mRNA expressions had increased tumour size, ER - and PR - tumours (all adjusted $p$ values $\leq 0.01)$. Interestingly, Genufu subtype (ER+/HER2-/low proliferation) and PAM50.Luminal A subtype were common in tumours with low MYC mRNA and high ATM mRNA expressions all adjusted $p$ values $\leq 0.01$ ). Her-2+ and PAM50.Her-2 subtypes were more common in tumours with low MYC mRNA and low ATM mRNA expressions (all adjusted $p$ values $\leq 0.01$ ). Whereas triple negative, Genefu subtype (ER-/Her-2-), PAM50.Basal were frequently expressing in tumours with high MYC mRNA and high ATM mRNA expressions (all adjusted $p$ values $\leq 0.01$ ).
We then investigated the prognostic significance of MYCATM co-expression in breast cancers. In the whole cohort, as shown in Fig. 2a, d, patients with MYC overexpressed tumours with low ATM protein or mRNA expression had worse overall breast cancer-specific survival (BCSS) $(p<0.001)$. In ER+ breast cancer, similarly, MYC overexpressed tumours with low ATM levels were associated with worse survival $(p<0.001)$ (Fig. 2b, e) including in patients who received endocrine therapy (Supplementary Figs. S3B and $\mathrm{S} 3 \mathrm{~F}$ ). In ER - tumours that received no chemotherapy, MYC overexpressed tumours with low ATM protein levels had the worst survival (Supplementary Fig. S3C). Whereas at the mRNA level, ER- tumours that received chemotherapy have poor survival $(p=0.028)$ (Supplementary Fig. $\mathrm{S} 3 \mathrm{H})$. Together, our data show that MYC-ATM co-expression has prognostic significance in breast cancers.

\section{Discussion}

Oxidative and oncogenic stresses in MYC overexpressed tumours will induce DNA damage. The DNA damage signalling proteins, ATR and ATM, are critical for the 
Table 3 ATM and MYC protein co-expression in sporadic breast cancer

\begin{tabular}{|c|c|c|c|c|c|c|}
\hline \multirow[t]{2}{*}{ Variable } & \multicolumn{4}{|c|}{ ATM and MYC protein co-expression } & \multirow[t]{2}{*}{$p$ value } & \multirow[t]{2}{*}{ Adjusted $p$ value* } \\
\hline & $\begin{array}{l}\text { MYC-/ATM- } \\
N(\%)\end{array}$ & $\begin{array}{l}\text { MYC-/ATM+ } \\
N(\%)\end{array}$ & $\begin{array}{l}\text { MYC+/ATM- } \\
N(\%)\end{array}$ & $\begin{array}{l}\text { MYC+/ATM+ } \\
N(\%)\end{array}$ & & \\
\hline \multicolumn{7}{|l|}{ Tumour size $(\mathrm{cm})$} \\
\hline$\leq 2.0$ & $93(45.8)$ & $77(54.2)$ & $66(41.0)$ & $96(50.5)$ & 0.101 & 1.616 \\
\hline$>2.0$ & $110(54.2)$ & $65(45.8)$ & $95(59.0)$ & $94(49.5)$ & & \\
\hline \multicolumn{7}{|l|}{ Vascular invasion } \\
\hline Negative & $127(62.9)$ & $112(78.9)$ & $103(64.0)$ & $129(68.3)$ & 0.01 & 0.013 \\
\hline Positive & $75(37.1)$ & $30(21.1)$ & $58(36.0)$ & $60(31.7)$ & & \\
\hline \multicolumn{7}{|l|}{ Tumour grade ${ }^{\mathrm{a}}$} \\
\hline G1 & $23(11.3)$ & $39(27.2)$ & $9(5.6)$ & $41(21.7)$ & $<0.00001$ & $<0.00001$ \\
\hline $\mathrm{G} 2$ & $61(30.0)$ & $54(37.8)$ & $48(29.8)$ & $60(31.7)$ & & \\
\hline G3 & $119(58.6)$ & $50(35.0)$ & $104(64.6)$ & $88(46.6)$ & & \\
\hline \multicolumn{7}{|l|}{ Tumour type } \\
\hline Ductal (incl mixed) & $172(84.7)$ & $117(82.4)$ & $140(87.0)$ & $169(89.0)$ & 0.023 & 0.028 \\
\hline Lobular & $17(8.5)$ & $18(12.7)$ & $10(6.2)$ & $9(4.7)$ & & \\
\hline Medullary-like & $7(3.4)$ & $0(0.0)$ & $9(5.6)$ & $8(4.2)$ & & \\
\hline Special type & $7(3.4)$ & $7(4.9)$ & $2(1.2)$ & $4(2.1)$ & & \\
\hline \multicolumn{7}{|l|}{ Tubules } \\
\hline 1 & $9(4.5)$ & $10(7.1)$ & $5(3.1)$ & $6(3.2)$ & 0.00016 & 0.00028 \\
\hline 2 & 49 (24.6) & $57(40.7)$ & 45 (28.7) & $83(44.9)$ & & \\
\hline 3 & $141(70.9)$ & $73(52.2)$ & $107(68.2)$ & $96(51.9)$ & & \\
\hline \multicolumn{7}{|l|}{ Pleomorphism } \\
\hline 1 & $4(2.0)$ & $4(2.8)$ & $1(0.7)$ & $6(3.3)$ & 0.00007 & 0.00015 \\
\hline 2 & $69(34.7)$ & $74(52.9)$ & $41(26.1)$ & 73 (39.9) & & \\
\hline 3 & $126(66.3)$ & $62(44.3)$ & $115(73.2)$ & $104(56.8)$ & & \\
\hline \multicolumn{7}{|l|}{ Mitosis } \\
\hline 1 & $58(29.1)$ & 75 (53.6) & $29(18.5)$ & $62(33.5)$ & $<0.00001$ & $<0.00001$ \\
\hline 2 & 35 (17.6) & $17(12.1)$ & $28(17.8)$ & $42(22.7)$ & & \\
\hline 3 & $106(53.3)$ & $48(34.3)$ & $100(63.7)$ & $81(43.8)$ & & \\
\hline \multicolumn{7}{|l|}{ NPI group } \\
\hline GPG & 48 (23.7) & $59(41.6)$ & $28(17.4)$ & $65(34.2)$ & $<0.00001$ & 0.00001 \\
\hline MPG & $118(58.1)$ & $76(53.5)$ & 97 (60.2) & $100(52.6)$ & & \\
\hline PPG & $37(18.2)$ & $7(4.9)$ & $36(22.4)$ & $25(13.2)$ & & \\
\hline \multicolumn{7}{|l|}{ ER status } \\
\hline Negative & $69(33.8)$ & $23(16.1)$ & 59 (36.6) & $40(21.1)$ & 0.00002 & 0.00007 \\
\hline Positive & $135(66.2)$ & $120(83.9)$ & $102(63.4)$ & $150(78.9)$ & & \\
\hline \multicolumn{7}{|l|}{ PR status } \\
\hline Negative & $100(49.8)$ & $41(29.1)$ & $83(51.6)$ & $65(34.4)$ & 0.00001 & 0.00006 \\
\hline Positive & $101(50.2)$ & $100(70.9)$ & $78(48.4)$ & $124(65.6)$ & & \\
\hline \multicolumn{7}{|l|}{ HER2 status } \\
\hline Negative & $163(81.5)$ & $127(91.4)$ & $136(85.0)$ & $158(83.2)$ & 0.081 & 0.086 \\
\hline Positive & 37 (18.5) & $12(8.6)$ & $24(15.0)$ & $32(16.8)$ & & \\
\hline \multicolumn{7}{|l|}{ Triple negative } \\
\hline Non-triple negative & $159(79.1)$ & $124(87.3)$ & $112(69.6)$ & $165(86.8)$ & 0.00007 & 0.00015 \\
\hline Triple negative & $42(20.9)$ & $18(12.7)$ & $49(30.4)$ & $25(13.2)$ & & \\
\hline \multicolumn{7}{|l|}{ Basal phenotype } \\
\hline Negative & $160(79.6)$ & $110(78.6)$ & $107(66.5)$ & $131(68.9)$ & 0.008 & 0.0116 \\
\hline Positive & $41(20.3)$ & $30(21.4)$ & $54(33.5)$ & $59(30.1)$ & & \\
\hline
\end{tabular}

Bold statistically significant; NPI Nottingham Prognostic Index, GPG Good Prognosis Group, MPG Moderate Prognosis Group, $P P G$ Poor Prognosis Group, HER2 Human epidermal growth factor 2, ER oestrogen receptor, PR progesterone receptor; Basal-like: ER-, HER2- and positive expression of either CK5/6, CK14, or EGFR; Triple negative: ER-/PR-/HER2-

*Adjusted $p$ value-Benjamini and Hochberg false discovery rate

${ }^{a}$ Grade as defined by Nottingham Grading System 
Table 4 ATM and MYC mRNA co-expression in sporadic breast cancer

\begin{tabular}{|c|c|c|c|c|c|c|}
\hline \multirow[t]{3}{*}{ Variable } & \multicolumn{4}{|c|}{$A T M$ and $M Y C$ mRNA co-expression } & \multirow[t]{3}{*}{$p$ value } & \multirow[t]{3}{*}{ Adjusted $p$ value* } \\
\hline & MYC-/ATM- & MYC-/ATM+ & MYC+/ATM- & MYC+/ATM+ & & \\
\hline & $N(\%)$ & $N(\%)$ & $N(\%)$ & $N(\%)$ & & \\
\hline \multicolumn{7}{|l|}{ Tumour size $(\mathrm{cm})$} \\
\hline $\mathrm{T} 1 \mathrm{a}+\mathrm{b}(1.0)$ & $6(3.3)$ & $76(5.0)$ & $3(16.7)$ & $7(3.1)$ & 0.00038 & 0.0009 \\
\hline $\mathrm{T} 1 \mathrm{c}(>1.0-2.0)$ & $72(39.3)$ & $611(39.9)$ & $2(11.1)$ & $81(36.2)$ & & \\
\hline $\mathrm{T} 2(>2.0-5)$ & $89(48.6)$ & $779(50.8)$ & $9(50.0)$ & $124(55.4)$ & & \\
\hline $\mathrm{T} 3(>5)$ & $16(8.7)$ & $66(4.3)$ & $4(22.2)$ & $12(5.4)$ & & \\
\hline \multicolumn{7}{|l|}{ Lymph node stage } \\
\hline Negative & $98(53.0)$ & $820(53.1)$ & $9(50.0)$ & $108(48.2)$ & 0.803 & \\
\hline Positive (1-3) & $32(17.3)$ & $243(15.7)$ & $2(11.1)$ & $37(16.5)$ & & \\
\hline Positive (>3) & $55(29.7)$ & $481(31.2)$ & $7(38.9)$ & $79(35.3)$ & & \\
\hline \multicolumn{7}{|l|}{ Grade $^{\mathrm{a}}$} \\
\hline G1 & $20(11.2)$ & $137(9.3)$ & $0(0.0)$ & $12(5.4)$ & 0.00006 & 0.0002 \\
\hline $\mathrm{G} 2$ & $73(40.8)$ & $627(4.6)$ & $8(47.1)$ & $62(28.1)$ & & \\
\hline G3 & $86(48.0)$ & $708(48.1)$ & $9(52.9)$ & $147(66.5)$ & & \\
\hline \multicolumn{7}{|l|}{ NPI } \\
\hline$\leq 3.4$ & $36(20.3)$ & $322(22.0)$ & $3(17.6)$ & $27(12.6)$ & 0.016 & 0.0288 \\
\hline$>3.4$ & $141(79.7)$ & $1139(78.0)$ & $14(82.4)$ & $188(87.4)$ & & \\
\hline \multicolumn{7}{|l|}{ HER 2 overexpression } \\
\hline No & $157(84.9)$ & $1344(86.9)$ & $17(94.4)$ & $214(94.3)$ & 0.007 & 0.01 \\
\hline Yes & $28(15.1)$ & $203(13.1)$ & $1(5.6)$ & $13(5.7)$ & & \\
\hline \multicolumn{7}{|l|}{ ER } \\
\hline Negative & $39(21.1)$ & 323 (20.9) & $6(33.3)$ & $102(44.9)$ & $<0.00001$ & $<0.00001$ \\
\hline Positive & $146(78.9)$ & $1224(79.1)$ & $12(66.7)$ & $125(55.1)$ & & \\
\hline \multicolumn{7}{|l|}{ PR } \\
\hline Negative & $76(41.1)$ & $715(46.2)$ & $14(77.8)$ & $131(57.7)$ & 0.00016 & 0.0004 \\
\hline Positive & $109(58.9)$ & $832(53.8)$ & $4(22.2)$ & $96(42.3)$ & & \\
\hline \multicolumn{7}{|l|}{ Triple negative } \\
\hline No & $162(87.6)$ & $1346(87.0)$ & $13(72.2)$ & $139(61.2)$ & $<0.00001$ & $<0.00001$ \\
\hline Yes & $23(12.4)$ & $201(13.0)$ & $5(27.8)$ & $88(38.8)$ & & \\
\hline \multicolumn{7}{|l|}{ Genefu subtype } \\
\hline ER-/Her-2 negative & $12(12.9)$ & $95(12.2)$ & $2(25.0)$ & $41(36.0)$ & $<0.00001$ & $<0.00001$ \\
\hline $\mathrm{ER}+/$ Her-2 negative/high proliferation & $33(35.5)$ & $285(36.6)$ & $3(37.5)$ & $45(39.5)$ & 0.933 & 25.191 \\
\hline $\mathrm{ER}+/$ Her-2 negative/low proliferation & $34(36.6)$ & $311(39.9)$ & $2(25.0)$ & $21(18.4)$ & 0.00015 & 0.0004 \\
\hline Her-2 positive & $14(15.1)$ & $88(11.3)$ & $1(12.5)$ & $7(6.1)$ & 0.224 & 0.288 \\
\hline \multicolumn{7}{|l|}{ PAM50 subtype } \\
\hline PAM50.Her2 & $31(18.3)$ & $201(14.3)$ & $1(7.1)$ & $5(2.7)$ & 0.00004 & 0.0001 \\
\hline PAM50.Basal & $23(13.6)$ & $204(14.5)$ & $6(42.9)$ & $97(52.4)$ & $<0.00001$ & $<0.00001$ \\
\hline PAM50.LumA & $67(39.6)$ & $608(43.3)$ & $2(14.3)$ & $38(20.5)$ & $<0.00001$ & $<0.00001$ \\
\hline PAM50.LumB & $48(28.4)$ & $391(27.8)$ & $5(35.7)$ & $45(24.3)$ & 0.67 & 0.7865 \\
\hline
\end{tabular}

Bold statistically significant; NPI Nottingham Prognostic Index, HER2 Human epidermal growth factor 2, ER oestrogen receptor, $P R$ progesterone receptor

*Adjusted $p$ value-Benjamini and Hochberg false discovery rate

${ }^{\mathrm{a}}$ Grade as defined by Nottingham Grading System 
maintenance of genomic instability [6, 15]. Although MYC amplification promotes aggressive breast cancer phenotype [7, 16, 21, 38], whether ATR and ATM expressions influence pathology and clinical outcomes in MYC overexpressed breast cancers is unknown.

MYC promotes cellular proliferation by several mechanisms, including by promoting replication and transcriptional response [5, 15, 17]. However, MYC overexpression also induces replication stress [17]. Activation of ATR-mediated signalling is a key compensatory response to mitigate replication stress in MYC overexpressed tumours [6]. Therefore, ATR overexpression in MYC overexpressed tumours will be expected to promote proliferation and aggressive phenotypes. In the current study, we provide the first clinical evidence that high ATR in MYC overexpressed tumours is associated with aggressive cancer and poor survival. Although direct targeting MYC for cancer therapy has been challenging $[9,26]$, the clinical data shown here would suggest that ATR-Chk1 pathway targeting could be an alternative anti-cancer approach in MYC-amplified breast cancers. A previous preclinical study investigating the role of Chk1 expression in MYC amplified tumours has in fact shown that its blockade resulted in caspase-depended apoptosis of the MYC-overexpressing tumours cells both in vitro and in murine models of B-cell lymphoma [22]. MYC is a wellknown ER-regulated gene and its overexpression is linked to resistance to endocrine therapy [7, 8, 21, 33, 36, 39]. In addition, MYC is frequently overexpressed during progression and distant relapse of ER+ breast cancers and predicts poor outcome following adjuvant endocrine treatment [21, 29]. In another study, MYC expression was up-regulated in aromatase inhibitor-resistant breast cancer cells and reduction of MYC expression significantly decreased cell proliferation in breast cancer cell lines [8]. In the current study, high ATR in MYC overexpressed tumours was linked to poor survival particularly in patients who received endocrine therapy providing further evidence for ATR as a predictive factor in MYC overexpressed ER+ breast cancers. As ATR inhibition is a promising anti-cancer approach $[18,24]$, whether combining ATR inhibitor with endocrine therapy in MYC amplified tumours will be clinically relevant will be an interesting area for future investigation.

Proficient ATM-mediated pathways act as robust anticancer barriers $[3,4,10,11,13]$. In contrast, ATM deficiency either in the germ-line or due to epigenetic mechanisms is well known to increase cancer risk and promote breast cancers [3, 4, 10, 11, 13]. For example, ATM has been shown to promote apoptosis and suppress tumorigenesis in response to MYC [31]. Therefore, ATM deficiency in MYC overexpressed breast cancer will be expected to promote aggressive breast cancers. As expected, in contrast to ATR, we observed that low ATM was linked to aggressive phenotypes in MYC overexpressed breast cancers, including in ER+ tumours. PARP [20] or ATR inhibition $[25,30]$ can induce synthetic lethality in ATMdeficient haematological malignancies. Therefore, it will be important to evaluate if a similar approach could be employed to personalise therapy in ATM-deficient MYC overexpressed breast cancers.

In conclusion, we provide strong clinical evidence that ATM signalling and ATR signalling can influence clinicopathological features and survival outcomes in patients with MYC overexpressed breast cancer.

Acknowledgements We thank the Nottingham Health Science Biobank and Breast Cancer Now Tissue Bank for the provision of tissue samples.

\section{Compliance with ethical standards}

Conflict of interest The authors declare no conflicts of interests.

Ethical approval All procedures performed in studies involving human participants were in accordance with the ethical standards of the institutional and/or national research committee and with the 1964 Helsinki declaration and its later amendments or comparable ethical standards.

Open Access This article is distributed under the terms of the Creative Commons Attribution 4.0 International License (http://creativeco mmons.org/licenses/by/4.0/), which permits unrestricted use, distribution, and reproduction in any medium, provided you give appropriate credit to the original author(s) and the source, provide a link to the Creative Commons license, and indicate if changes were made.

\section{References}

1. Abdel-Fatah TM et al (2014) Clinicopathological significance of ATM-Chk2 expression in sporadic breast cancers: a comprehensive analysis in large cohorts. Neoplasia 16:982-991. https://doi. org/10.1016/j.neo.2014.09.009

2. Abdel-Fatah TM et al (2015) Untangling the ATR-CHEK1 network for prognostication, prediction and therapeutic target validation in breast cancer. Mol Oncol 9:569-585. https://doi. org/10.1016/j.molonc.2014.10.013

3. Ahmed M, Rahman N (2006) ATM and breast cancer susceptibility. Oncogene 25:5906-5911. https://doi.org/10.1038/ sj.onc. 1209873

4. Boohaker RJ, Xu B (2014) The versatile functions of ATM kinase. Biomed J 37:3-9. https://doi.org/10.4103/2319-4170.125655

5. Bretones G et al (2015) Myc and cell cycle control. Biochim Biophys Acta 1849:506-516. https://doi.org/10.1016/j.bbagr m.2014.03.013

6. Campaner S, Amati B (2012) Two sides of the Myc-induced DNA damage response: from tumor suppression to tumor maintenance. Cell Div 7:6-6. https://doi.org/10.1186/1747-1028-7-6

7. Chen Y, Olopade OI (2008) MYC in breast tumor progression. Expert Rev Anticancer Ther 8:1689-1698. https://doi. org/10.1586/14737140.8.10.1689

8. Chen $\mathrm{Z}$ et al (2015) Cross-talk between ER and HER2 regulates c-MYC-mediated glutamine metabolism in aromatase inhibitor 
resistant breast cancer cells. J Steroid Biochem Mol Biol 149:118127. https://doi.org/10.1016/j.jsbmb.2015.02.004

9. Chen $\mathrm{H}$ et al (2018) Targeting oncogenic Myc as a strategy for cancer treatment. Signal Transduct Target Ther 3:5. https://doi. org/10.1038/s41392-018-0008-7

10. Choi $\mathrm{M}$ et al (2016) ATM mutations in cancer: therapeutic implications. Mol Cancer Ther 15:1781-1791. https://doi. org/10.1158/1535-7163.MCT-15-0945

11. Clouaire T et al (2017) Taming tricky DSBs: ATM on duty. DNA Repair 56:84-91. https://doi.org/10.1016/j.dnarep.2017.06.010

12. Conacci-Sorrell M et al (2014) An overview of MYC and its interactome. Cold Spring Harb Perspect Med 4:a014357. https://doi. org/10.1101/cshperspect.a014357

13. Cremona CA, Behrens A (2014) ATM signalling and cancer. Oncogene 33:3351-3360. https://doi.org/10.1038/onc.2013.275

14. Curtis $C$ et al (2012) The genomic and transcriptomic architecture of 2,000 breast tumours reveals novel subgroups. Nature 486:346352. https://doi.org/10.1038/nature10983

15. Dang CV (2012) MYC on the path to cancer. Cell 149:22-35. https://doi.org/10.1016/j.cell.2012.03.003

16. Deming SL et al (2000) C-myc amplification in breast cancer: a meta-analysis of its occurrence and prognostic relevance. Br J Cancer 83:1688-1695. https://doi.org/10.1054/bjoc.2000.1522

17. Dominguez-Sola D, Gautier J (2014) MYC and the control of DNA replication. Cold Spring Harb Perspect Med 4:a014423. https://doi.org/10.1101/cshperspect.a014423

18. Fokas E et al. Targeting ATR in DNA damage response and cancer therapeutics. Cancer Treatment Reviews 40:109-117. https://doi. org/10.1016/j.ctrv.2013.03.002

19. Gabay M et al (2014) MYC activation is a hallmark of cancer initiation and maintenance. Cold Spring Harb Perspect Med 4:a014241. https://doi.org/10.1101/cshperspect.a014241

20. Gilardini Montani MS et al (2013) ATM-depletion in breast cancer cells confers sensitivity to PARP inhibition. J Exp Clin Cancer Res 32:95. https://doi.org/10.1186/1756-9966-32-95

21. Green AR et al (2016) MYC functions are specific in biological subtypes of breast cancer and confers resistance to endocrine therapy in luminal tumours. Br J Cancer 114:917-928. https://doi. org/10.1038/bjc.2016.46

22. Hoglund A et al (2011) Therapeutic implications for the induced levels of Chk1 in Myc-expressing cancer cells. Clin Cancer Res 17:7067-7079. https://doi.org/10.1158/1078-0432.ccr-11-1198

23. Holm S (1979) A simple sequentially rejective multiple test procedure. Scand J Stat 1979 6:65-70

24. Karnitz LM, Zou L (2015) Molecular pathways: targeting ATR in cancer therapy. Clin Cancer Res 21:4780-4785. https://doi. org/10.1158/1078-0432.ccr-15-0479
25. Kwok M et al (2016) ATR inhibition induces synthetic lethality and overcomes chemoresistance in TP53- or ATM-defective chronic lymphocytic leukemia cells. Blood 127:582-595. https:// doi.org/10.1182/blood-2015-05-644872

26. McKeown MR, Bradner JE (2014) Therapeutic strategies to inhibit MYC. Cold Spring Harb Perspect Med 4:a014266. https ://doi.org/10.1101/cshperspect.a014266

27. McMahon SB (2014) MYC and the control of apoptosis. Cold Spring Harb Perspect Med 4:a014407. https://doi.org/10.1101/ cshperspect.a014407

28. McShane LM et al (2005) Reporting recommendations for tumor marker prognostic studies (REMARK). J Natl Cancer Inst 97:1180-1184. https://doi.org/10.1093/jnci/dji237

29. Miller TW et al (2011) A gene expression signature from human breast cancer cells with acquired hormone independence identifies MYC as a mediator of antiestrogen resistance. Clin Cancer Res 17:2024-2034. https://doi.org/10.1158/1078-0432.ccr-10-2567

30. Min A et al (2017) AZD6738, a novel oral inhibitor of ATR, induces synthetic lethality with ATM deficiency in gastric cancer cells. Mol Cancer Ther 16:566-577. https://doi.org/10.1158/15357163.MCT-16-0378

31. Pusapati RV et al (2006) ATM promotes apoptosis and suppresses tumorigenesis in response to Myc. Proc Natl Acad Sci USA 103:1446-1451. https://doi.org/10.1073/pnas.0507367103

32. Saldivar JC et al (2017) The essential kinase ATR: ensuring faithful duplication of a challenging genome. Nat Rev Mol Cell Biol 18:622. https://doi.org/10.1038/nrm.2017.67

33. Shang Y et al (2000) Cofactor dynamics and sufficiency in estrogen receptor-regulated transcription. Cell 103:843-852

34. Shiloh Y, Ziv Y (2013) The ATM protein kinase: regulating the cellular response to genotoxic stress, and more. Nat Rev Mol Cell Biol 14:197. https://doi.org/10.1038/nrm3546

35. Soucek L, Evan GI (2010) The ups and downs of Myc biology. Curr Opin Genet Dev 20:91-95. https://doi.org/10.1016/j. gde.2009.11.001

36. Wang C et al (2011) Estrogen induces c-myc gene expression via an upstream enhancer activated by the estrogen receptor and the AP-1 transcription factor. Mol Endocrinol 25:1527-1538. https:// doi.org/10.1210/me.2011-1037

37. Wolfer A, Ramaswamy S (2011) MYC and metastasis. Cancer Res 71:2034-2037. https://doi.org/10.1158/0008-5472.CAN-10-3776

38. Xu J et al (2010) MYC and breast cancer. Genes Cancer 1:629640. https://doi.org/10.1177/1947601910378691

39. Zhang $\mathrm{Y}$ et al (2016) Targeting radioresistant breast cancer cells by single agent $\mathrm{CHK} 1$ inhibitor via enhancing replication stress. Oncotarget 7:34688-34702. https://doi.org/10.18632/oncotarget .9156 